Annuaire suisse de politique de développement

25-1 | 2006

Faits et statistiques 2006

\title{
10. Environnement et développement
}

\author{
Xavier Tschumi Canosa
}

\section{OpenEdition}

Journals

Édition électronique

URL : http://journals.openedition.org/aspd/322

DOI : 10.4000/aspd.322

ISSN : 1663-9669

\section{Éditeur}

Institut de hautes études internationales et du développement

\section{Édition imprimée}

Date de publication : 1 avril 2006

Pagination : 157-174

ISBN : 2-88247-061-4

ISSN : 1660-5934

Référence électronique

Xavier Tschumi Canosa, «10. Environnement et développement », Annuaire suisse de politique de développement [En ligne], 25-1 | 2006, mis en ligne le 12 février 2010, consulté le 08 septembre 2020. URL : http://journals.openedition.org/aspd/322 ; DOI : https://doi.org/10.4000/aspd.322 


\section{Environnement et développement*}

10.1. Développement durable

L'ORGANISATION des Nations unies pour l'éducation, la science et la culture (UNESCO) a lancé en 2005 la Décennie pour l'éducation en vue du développement durable, dont le but est d'intégrer les principes, les valeurs et les pratiques $d u$ développement durable dans tous les aspects de la formation. Ce travail de fond vient compléter les stratégies plus spécifiques de développement durable mises en place aux niveaux international, national et local. Le premier cycle biennal de sessions de la Commission du développement durable s'est terminé en avril 2005. Il portait sur l'eau, l'assainissement et les établissements humains et a débouché sur des engagements politiques, qui, selon l'évaluation de la Suisse, permettent de passer à l'action.

La mise en cuvre locale du développement durable est un processus en cours en Suisse et c'est pour en mesurer l'avancement comparatif et, le cas échéant, en corriger la direction que ce pays a mis au point, en 2005, un système d'indicateurs au niveau de cantons et de communes pilotes.

\subsubsection{Décennie des Nations unies pour l'éducation en vue du développement durable 2005-2014}

Le concept de développement durable s'est imposé depuis le Sommet de Rio en 1992, mais bien qu'ayant été adopté très largement au niveau stratégique par les Etats pour leur propre développement ou leur aide au développement, il peine à entrer dans les pratiques quotidiennes des populations. Leur éducation au développement durable doit donc être renforcée.

La proposition d'instaurer une Décennie des Nations unies pour l'éducation en vue du développement durable 2005-2014 a émané du Sommet mondial de Johannesburg pour le développement durable en septembre 2002, et c'est l'Assemblée générale des Nations unies qui, dans une résolution ${ }^{1}$, en a pris la décision en décembre de cette même année.

Dans cette résolution, l'UNESCO a été désignée comme organe responsable de la promotion de la décennie et chargée d'en préparer un programme d'application international. Cette dernière a été lancée officiellement le $1^{\text {er }}$ mars 2005 et le plan international de sa mise en œuvre adopté par le Conseil exécutif de l'UNESCO le 11 août 2005, après près de deux ans de consultations d'abord au sein du système des Nations unies, puis au niveau mondial auprès des gouvernements, des ONG et des universités. «Le but global de la décennie est d'intégrer les principes, les valeurs et les pratiques du développement durable dans tous les

* Par Xavier Tschumi Canosa, collaborateur scientifique à l'IUED.

1 Assemblée générale des Nations unies, résolution 57/254 du 20 décembre 2002, doc. A/RES/ $57 / 254$. 
aspects de l'éducation et de l'apprentissage. $»^{2}$ L'UNESCO peut compter sur ses commissions nationales basées dans la plupart de ses pays membres, au nombre de 191 en mars 2005, pour mener à bien son plan de mise en œuvre.

La Commission suisse pour l'UNESCO a mis en place un comité d'experts pour donner à cette décennie un profil clair et un contenu solide en Suisse. Ce comité a, dans un premier temps, défini ses buts stratégiques ${ }^{3}$, qui consiste à renforcer les efforts de formation formelle et informelle dans le domaine du développement durable et à soutenir, avec les moyens politiques et financiers disponibles, les acteurs de l'éducation au développement durable ainsi que les projets qu'ils mettront en place dans le cadre de la décennie.

La Commission suisse pour l'UNESCO a lancé un site Internet consacré à la décennie et à sa mise en œuvre ${ }^{4}$, en Suisse particulièrement mais également au niveau mondial. En effet, chaque commission nationale, dans la mesure de ses capacités, pourra également créer un tel site, basé sur le modèle défini par l'UNESCO.

La commission a également patronné un forum national sur l'éducation au développement durable, organisé le 29 janvier 2005 à Genève par l'association GEDUC (Global/General Education). Ce forum national a réuni plus de 280 personnes - enseignants, politiciens, journalistes - et s'est concentré sur l'éducation citoyenne, le rôle des universités et celui des médias, la formation des jeunes et la formation continue. Il visait à favoriser le partage d'informations et d'expériences en matière d'éducation au développement durable et à encourager les partenariats et initiatives en vue de satisfaire les objectifs de la décennie.

L' «intégration du concept de développement durable au sein de l'école et dans l'enseignement $»^{5}$ figure aussi parmi les objectifs que s'est fixés la Conférence suisse des directeurs cantonaux de l'instruction publique dans son programme annuel de travail, adopté en août 2005. Dans ce projet, la priorité ira aux thèmes de la santé, du développement et de l'environnement.

\subsubsection{3e Session de la Commission des Nations unies pour le développement durable (New York, 11-22 avril 2005)}

La 13e Session de la Commission pour le développement durable (CDD-13) s'est déroulée du 11 au 22 avril 2005 à New York. Conformément au programme de travail pluriannuel adopté en 2003, les sessions de la CDD sont organisées, depuis 2004, en cycles biennaux portant chacun sur des thèmes distincts.

An Annuaire 2005, $\mathrm{n}^{\circ}$ 1, cycles biennaux de travail de la CDD, pp. 154-155.

La CDD-13 a donc porté sur les mêmes thématiques que la session d'évaluation précédente en 2004, soit l'eau, l'assainissement et les établissements humains,

2 UNESCO, Rapport du directeur général sur la Décennie des Nations unies pour l'éducation au service du développement durable: Plan international de mise en auvre et contribution de l'UNESCO à la mise en œuvre de la décennie, Paris, 11 août 2005, doc. 172 EX/11, Annexe I - p. 2.

3 Thomas Baumann, «Schweizerische UNESCO-Kommission. Aufgaben und Ziele für die Dekade $\mathrm{BNE}$ », ph akzente, $\mathrm{n}^{\circ}$ 3, 2005, pp. 12-13.

4 <www.unesco-developpement-durable.ch>.

5 Conférence suisse des directeurs cantonaux de l'instruction publique, Programme de travail de la CDIP, 16 juin 2005, p. 7, disponible sur le site Internet de la CDIP, <www.cdip.ch>. 
mais a bâti sur les résultats de cette dernière pour se focaliser sur les mesures politiques propres à concrétiser les engagements pris dans le cadre du Plan de mise en œuvre de Johannesburg (JPOI) et des Objectifs du Millénaire pour le développement (OMD). Comme la CDD-13 concluait le tout premier cycle biennal de négociations, elle établissait un précédent pour les futures sessions et avait aussi valeur de test de la volonté politique internationale dans le domaine du développement durable, et notamment en ce qui concerne le financement des mesures décidées.

Vu les thématiques traitées, c'est un représentant de l'Office fédéral de la santé publique qui a conduit la délégation suisse à la CDD-13. Pour le prochain cycle biennal de sessions de la CDD, en 2006-2007, c'est la DDC qui sera le chef de file ${ }^{6}$.

Les sources de financement ont été un des principaux points négociés à la CDD13, les autres ayant été l'engagement des acteurs (notamment la société civile et le secteur privé), le recouvrement des coûts et les subsides, ainsi que l'approche écosystémique. Le document conclusif de la CDD-13 (la Décision), négocié et adopté par consensus au niveau ministériel par les Etats parties, fournit les lignes directrices que ces Etats s'engagent à suivre dans les trois thématiques du cycle. D'une manière générale, la Suisse s'estime satisfaite du résultat de la CDD-13. Les points qu'elle avait défendus sont généralement repris dans le texte de la Décision de la CDD-13 et celle-ci est jugée suffisamment concrète pour passer à l'action.

En ce qui concerne l'eau, les engagements concourent à la réalisation de l'objectif du Millénaire pour le développement relatif à cette thématique ${ }^{7}$ et portent avant tout sur l'accès aux services d'approvisionnement en eau et sur la gestion intégrée des ressources en eau. Pour chacun de ces volets, deux exigences principales sont formulées dans la Décision: l'augmentation du financement, dont l'APD, et le renforcement des technologies et des capacités en ressources humaines qui doivent les accompagner. C'est dans cette thématique de l'eau douce que la Suisse s'est le plus engagée, notamment sur l'implication accrue du secteur privé en faveur d'un meilleur accès aux services d'approvisionnement en eau. Une autre priorité défendue par la Suisse dans cette thématique était l'indissociabilité de l'accès des pauvres à l'eau et de la protection et de l'utilisation durable des écosystèmes, ceux-ci étant intrinsèquement liés au cycle de l'eau.

En ce qui concerne les thématiques de l'assainissement et de l'hygiène, les engagements se focalisent sur le rôle que celles-ci peuvent jouer dans la réduction de la pauvreté et sur les mesures tenant compte d'une approche sensible au genre, notamment dans les écoles, les lieux de travail et les centres de santé. La nécessité d'élargir et d'augmenter les sources de financement spécifiquement dédiés à l'assainissement est aussi recommandée par ces directives. La Suisse a défendu l'importance de l'assainissement individuel et de l'hygiène individuelle, en

6 Ce cycle portera sur les thèmes suivants : énergie, atmosphère et pollution, développement industriel et changements climatiques.

7 Objectif 7: Assurer un environnement durable: réduire de moitié le pourcentage de la population qui n'a pas accès de façon durable à un approvisionnement en eau potable (pour la liste complète des OMD, voir, dans cet Annuaire, la section 2.1 du chapitre 2). 
termes d'économie et de santé, et des campagnes publiques de sensibilisation dans ce sens. Pour la Suisse, les mesures visant à améliorer l'assainissement doivent s'articuler avec les projets d'eau potable, pour profiter des synergies possibles entre ces deux thématiques.

En ce qui concerne les établissements humains, les engagements visent à prévenir l'apparition de nouveaux taudis par la planification et la gestion intégrée. C'est ONU-HABITAT (Programme des Nations unies pour les établissements humains) qui a été désigné pour suivre les mesures mises en œuvre dans le cadre de cette thématique, alors que c'est la CDD elle-même qui assurera le suivi dans les deux autres thématiques.

\subsubsection{Activités en Suisse pour le développement durable}

La politique de développement durable en Suisse, dont la mise en œuvre est assurée par la Confédération, les cantons et les communes, est guidée par la Stratégie 2002 du Conseil fédéral ${ }^{8}$.

DD Annuaire 2003, $\mathrm{n}^{\circ}$ 1, développement durable en Suisse, pp. 160-166.

Au niveau de la Confédération, c'est l'Office fédéral du développement territorial (ARE) qui est chargé de coordonner cette politique, laquelle implique une vingtaine de services fédéraux regroupés au sein du Comité interdépartemental pour le développement durable (CIDD) ${ }^{9}$. L'organe de pilotage de ce comité, dénommé Bureau du CIDD, est constitué de cinq services fédéraux: 1'ARE, la Direction du développement et de la coopération (DDC), le Secrétariat d'Etat à l'économie (seco), l'Office fédéral de l'environnement (OFEV) et l'Office fédéral de la santé publique (OFSP).

En matière d'Agendas 21, c'est au travers du Forum du développement durable, créé en juin 2001, que l'ARE coopère avec les cantons et les villes qui les mettent en œuvre à leurs niveaux respectifs. En décembre 2005, 139 communes et 14 cantons avaient engagé des démarches de développement durable, que ce soit dans le cadre de tels Agendas ou non. L'état des lieux de ces démarches est publié et régulièrement mis à jour par l'ARE ${ }^{10}$.

[DA Annuaire 2005, $\mathrm{n}^{\circ}$ 1, promotion d'Agendas 21 locaux, pp. 153-154.

En 2005, l'ARE a publié le rapport du Cercle Indicateurs ${ }^{11}$, qui est une plateforme nationale rattachée au Forum du développement durable et dont le but principal est de définir deux systèmes d'indicateurs centraux du développement durable, l'un pour les cantons et l'autre pour les villes qui font partie de ce cercle. Ce rapport est le fruit d'un projet lancé en 2003 et auquel ont pris part 14 villes et 8 cantons. Par «indicateurs centraux du développement durable» il faut entendre les indicateurs qui peuvent être mesurés aussi bien par les villes que par les cantons et qui permettent une évaluation comparative de leurs démarches

Conseil fédéral, Stratégie 2002 pour le développement durable (FF 2002 3678).

Nouvelle dénomination depuis octobre 2004. Ce comité s'intitulait auparavant Comité interdépartemental de Rio (Ci-Rio).

10 Sur le site Internet de l'ARE, <www.are.admin.ch> >développement durable >cantons et communes - agenda 21 local $>$ faits et chiffres.

11 Cercle Indicateurs, Indicateurs centraux pour le développement durable des villes et cantons, Berne, 30 octobre 2005, 88 p., disponible sur le site Internet de l'ARE, <www.are.admin.ch>. 
respectives (benchmarking et ultérieurement monitoring). En 2003, le système d'indicateurs MONET ${ }^{12}$ avait quant à lui permis de situer, sur un axe temporel, l'avancement de la Suisse au niveau national sur la voie de son développement durable (monitoring).

Le système d'indicateurs centraux des démarches cantonales et communales s'inscrit, de façon équilibrée, dans les trois dimensions du développement durable, l'environnement, l'économie et la société. Dans chacune de ces thématiques, une dizaine d'indicateurs centraux ont été choisis selon divers critères, puis mesurés. S'agissant des premiers résultats, seul le benchmarking entre les cantons et entre les villes a été possible. Les résultats ${ }^{13}$ sont donnés par indicateur, mais ont également été agrégés par thématique et pour le développement durable dans son ensemble. L'agrégation des résultats a d'ailleurs fait l'objet de critiques relatives au déficit de rigueur scientifique d'une telle démarche ${ }^{14}$. Indépendamment de ces classements, le projet a permis de concrétiser le concept de développement durable et d'orienter les démarches selon les faiblesses et les forces que ces indicateurs ont fait ressortir pour chacun des participants au cercle. La prochaine évaluation aura lieu en 2009.

\section{SOURCES}

UNESCO, Rapport du directeur général sur la Décennie des Nations unies pour l'éducation au service du développement durable: Plan international de mise en æuvve et contribution de l'UNESCO à la mise en œuvre de la décennie, Paris, 11 août 2005, doc. 172 EX/11, 33 p.

Cercle Indicateurs, Indicateurs centraux pour le développement durable des villes et cantons, Berne, 30 octobre 2005, 88 p., disponible sur le site Internet de l'ARE, <www.are.admin.ch>.

\subsection{Convention-cadre des Nations unies sur les changements climatiques (CCNUCC)}

Un pas historique a été franchi en 2005 concernant la lutte contre les changements climatiques. Le Protocole de Kyoto est entré en vigueur et a été rendu opérationnel par ses Etats parties. Dans les négociations internationales, la question principale est de savoir comment faire participer le plus vite possible tous les grands émetteurs de gaz à effet de serre à ce processus, les pays industrialisés non encore parties, comme les Etats-Unis, mais également les grands pays en développement comme la Chine, l'Inde et le Brésil dans le cadre de la deuxième période d'engagement, après 2012, lorsque la première période d'engagement du protocole aura pris fin. En Suisse, la politique climatique repose essentiellement sur la Loi sur le $\mathrm{CO}_{2}$, laquelle fixe des objectifs de réduction des émissions de gaz à effet de serre correspondant aux engagements pris dans le cadre du Protocole de Kyoto. Les projections montrent que les mesures volontaires de l'économie pour réduire de telles émissions ne permettront pas de réaliser ces objectifs et une taxe sur les combustibles a alors été proposée par le

12 Pour plus de détails, voir <www.monet.admin.ch> (hébergé sur le site de l'Office fédéral de la statistique).

13 Les états des lieux dans les villes et dans les cantons du Cercle Indicateurs sont disponibles sur le site Internet de l'ARE, <www.are.admin.ch> > développement durable > mesurer la durabilité >cantons et villes.

14 Les cantons de Genève et Vaud ainsi que la Ville de Lausanne ont refusé de figurer au classement agrégé. 
Conseil fédéral en 2005. En ce qui concerne les carburants, un délai de deux ans est accordé au centime climatique pour faire la preuve de son efficacité. En cas d'échec, une taxe sera également appliquée aux carburants.

\subsection{1. $11^{\mathrm{e}}$ Conférence des parties à la CCNUCC et $1^{\text {re }}$ Réunion des parties au Protocole de Kyoto (Montréal, 28 novembre-9 décembre 2005)}

La $11^{\mathrm{e}}$ Conférence des parties (COP-11) à la Convention-cadre des Nations unies sur les changements climatiques s'est déroulée à Montréal du 28 novembre au 9 décembre 2005. La Suisse y était représentée, au niveau ministériel, par Bruno Oberle, qui a remplacé Philippe Roch à la tête de l'Office fédéral de l'environnement, des forêts et du paysage (OFEFP) $)^{15}$ le $1^{\text {er }}$ octobre 2005.

Cette conférence avait un caractère historique dans le sens qu'elle a servi de cadre à la $1^{\text {re }}$ Réunion des parties au Protocole de Kyoto (COP/MOP-1), ce dernier étant entré en vigueur le 16 février 2005 après sa ratification par la Russie le 18 octobre 2004.

Cette première réunion du Protocole de Kyoto a permis d'adopter formellement les modalités de mise en œuvre du protocole telles qu'elles avaient été recommandées notamment dans les Accords de Marrakech ${ }^{16}$, le rendant ainsi totalement opérationnel. Les mécanismes flexibles ${ }^{17}$ prévus par le protocole peuvent dès lors être lancés ou renforcés, garantissant «un marché durable et efficace du carbone $»^{18}$, selon le chef du Secrétariat des changements climatiques. Le régime de conformité prévu par ces accords a également été adopté lors de la réunion, assurant que les Etats parties au protocole imputent tous de la même manière leurs émissions de gaz à effet de serre et sont sanctionnés en cas de non-respect des dispositions.

Le Protocole de Kyoto oblige ses Etats parties jusqu'en 2012 seulement et ceuxci ont discuté, à Montréal, des engagements à prendre pour les années suivantes. Pour préparer cette discussion, un séminaire d'experts gouvernementaux avait été organisé sur ce thème en mai 2005 à Bonn. La Suisse y avait défendu l'idée d'un régime international promouvant la réduction réelle et substantielle des émissions de gaz à effet de serre à une échelle globale ${ }^{19}$, auquel devraient participer les émetteurs principaux, y compris les grands pays en développement (Inde, Chine et Brésil surtout). Un groupe de travail a été mis en place lors de la

15 Dès le $1^{\text {er }}$ janvier 2006, cet office sera restructuré et renommé Office fédéral de l'environnement (OFEV).

16 Adoptés lors de la $7^{\text {e }}$ Conférence des parties à la convention en novembre 2001.

17 Renforcé lors de la réunion, le Mécanisme de développement propre permet aux pays devant réduire leurs émissions de gaz à effet de serre d'obtenir des certificats d'émission en finançant des projets de développement durable dans les pays en développement non soumis à l'obligation de réduction de leurs émissions. Lancé lors de la réunion de Montréal, le Mécanisme de mise en œuvre conjointe repose quant à lui sur le financement de projets permettant de réduire les émissions de gaz à effet de serre contre des certificats d'émission, mais se déroule uniquement entre les pays devant réduire leurs émissions.

18 Secrétariat de la CCNUCC, La Conférence des Nations unies sur les changements climatiques s'entend sur des mesures cruciales pour contrer les changements climatiques, communiqué de presse, 10 décembre 2005.

19 Switzerland: Summary of the Presentation to the Seminar of Government Experts, Bonn, 16-17 May 2005. Ce document, de même que la présentation complète de la Suisse, est disponible sur le site Internet de la convention-cadre, <www.unfecc.int>. 
conférence de Montréal, pour assurer autant que possible la continuité des périodes d'engagement sous le protocole. Les pays en développement ont admis la responsabilité commune de tous les pays dans les changements climatiques mais ont insisté sur le fait que celle-ci doit être différenciée pour eux. De leur côté, les principaux pays industrialisés ayant actuellement des obligations de réduction de leurs émissions sous le protocole ont partagé l'idée de la Suisse sur la participation des grands pays en développement aux efforts de réduction des émissions de gaz à effet de serre. Selon les prévisions, les pays en développement dans leur ensemble émettront autant de gaz à effet de serre que les pays industrialisés d'ici à 2015.

Lors de la conférence également, les Etats parties se sont entendus pour entraîner non seulement les grands pays en développement dans les efforts internationaux de réduction des émissions de gaz à effet de serre après 2012, mais également les autres grands émetteurs, les Etats-Unis en tête, le plus rapidement possible.

En ce qui concerne la convention elle-même, les Etats parties ont adopté un programme de travail sur les mesures d'adaptation aux changements climatiques ${ }^{20}$, particulièrement pour les pays les moins avancés et les petits Etats insulaires en développement. Ce programme doit leur permettre d'améliorer leur compréhension de leur vulnérabilité et de renforcer leur capacité d'adaptation. La Suisse estime que les effets des changements climatiques ne sont plus seulement circonscrits à ces pays et qu'il est nécessaire, pour elle, non seulement de les atténuer en réduisant ses émissions, mais aussi de s'y adapter ${ }^{21}$.

En outre, la Suisse a présenté sa quatrième communication nationale dans le cadre de la Convention-cadre et sa première communication nationale en application du protocole lors de la conférence ${ }^{22}$. Ce document recense les activités entreprises par la Suisse pour remplir ses engagements internationaux depuis la dernière communication nationale en novembre 2001. En plus de la Loi sur le $\mathrm{CO}_{2}$ (voir plus bas), les politiques et mesures pouvant contribuer à abaisser les émissions de gaz à effet de serre y sont exposées, dans les domaines de l'environnement, de l'énergie, des transports, de l'agriculture, des forêts et des déchets. La communication nationale présente aussi des projections d'émissions jusqu'en 2020 ainsi que l'analyse des incidences des changements climatiques sur la Suisse, l'évaluation de sa vulnérabilité et les mesures d'adaptation qu'elle est en train de ou qu'elle compte mettre en place.

\subsubsection{Loi sur le $\mathrm{CO}_{2}$ : décision du Conseil fédéral}

La Loi sur le $\mathrm{CO}_{2}$, entrée en vigueur le $1^{\mathrm{er}}$ mai 2000 , vise à réduire les émissions de $\mathrm{CO}_{2}$ des carburants de $8 \%$ et celles des combustibles de $15 \%$ à l'horizon

20 Programme de travail de Buenos Aires adopté lors de la $10^{\text {e }}$ Conférence des parties en 2004 (voir Annuaire suisse de politique de développement 2005, $\mathrm{n}^{\circ} 1$, p. 157).

21 OFEFP, Conférence sur le climat à Montréal: l'avenir de la politique climatique mondiale au centre des discussions, communiqué de presse, 23 novembre 2005.

22 Swiss Confederation, Switzerland's Fourth National Communication under the UNFCCC: First National Communication under the Kyoto Protocol to the UNFCCC, 2005, 238 p. Une synthèse en français de ce document est disponible sur le site Internet de l'Office fédéral de l'environnement, $<$ www.environnement-suisse.ch $>$. 
2008-2012 par rapport à leurs niveaux de 1990. Si ces objectifs sont atteints, la Suisse aura alors respecté ses engagements pris dans le cadre du Protocole de Kyoto (voir plus haut).

En octobre 2004, le Conseil fédéral a lancé une procédure de consultation destinée à révéler les préférences entre quatre variantes ${ }^{23}$, dont la mise en œuvre compléterait les mesures librement consenties déjà engagées. C'est d'ailleurs parce que, selon les prévisions, ces dernières ne permettaient plus, à elles seules, d'atteindre les objectifs de la loi que ces variantes ont été proposées.

Annuaire 2005, $\mathrm{n}^{\circ}$ 1, Loi sur le $\mathrm{CO}_{2}$ et procédure de consultation sur les quatre variantes, pp. 159-160.

Les résultats de cette procédure de consultation montrent que la taxe sur les émissions de $\mathrm{CO}_{2}$ engendrées par les combustibles est généralement soutenue, mais qu'aucune majorité claire ne se dessine pour les carburants, ni en faveur de la taxe sur les émissions de $\mathrm{CO}_{2}$, ni en faveur du centime climatique ${ }^{24}$. Sur cette base, le Conseil fédéral a décidé, le 23 mars 2005, de prélever une taxe sur le $\mathrm{CO}_{2}$ applicable aux combustibles et d'autoriser le prélèvement à l'essai d'un centime climatique sur les carburants (troisième variante légèrement modifiée).

Le 22 juin 2005, le Conseil fédéral a soumis au Parlement son Message concernant l'approbation du montant de la taxe sur le $\mathrm{CO}_{2}$ appliquée aux combustibles $^{25}$. Le montant proposé, 35 francs par tonne de $\mathrm{CO}_{2}$, a été calculé de telle manière à combler l'écart qui sépare, à l'horizon 2010, les prévisions de réduction des émissions de $\mathrm{CO}_{2}$ de l'objectif fixé pour les combustibles. Ces prévisions sont calculées et régulièrement actualisées par l'Office fédéral de l'énergie $(\mathrm{OFEN})^{26}$. La Commission de l'environnement du Conseil national est entrée en matière sur le projet du Conseil fédéral le 8 novembre 2005 mais a reporté ses discussions sur son contenu à janvier 2006, car le Parlement peut, en vertu de la Loi sur le $\mathrm{CO}_{2}$, modifier le montant de la taxe et non pas seulement adopter ou rejeter la proposition soumise par le Conseil fédéral. En décembre 2005, l'industrie, les petites et moyennes entreprises et les propriétaires fonciers ont proposé de remplacer la taxe sur le $\mathrm{CO}_{2}$ appliquée aux combustibles par un centime climatique comme pour les carburants (voir plus bas), estimant que le haut niveau du cours du pétrole devrait induire l'effet incitateur voulu par la taxe.

Le centime climatique sur les carburants constitue une mesure librement consentie du secteur pétrolier suisse pour éviter la taxe sur le $\mathrm{CO}_{2}$. Le Conseil fédéral a permis la mise à l'essai de cette mesure pour une durée de deux ans, au

23 Taxe sur le $\mathrm{CO}_{2}$ prélevée sur les combustibles et les carburants (deux variantes proposées); taxe sur les combustibles et centime climatique sur les carburants (troisième variante); centime climatique sur les carburants seul (quatrième variante).

24 Département fédéral de l'environnement, des transport, de l'énergie et de la communication (DETEC), Analyse des résultats de la procédure de consultation sur les mesures requises pour atteindre les objectifs de réduction des émissions fixés dans la Loi sur le $\mathrm{CO}_{2}$, Berne, 23 mars 2005, $31 \mathrm{p}$.

25 Conseil fédéral, Message concernant l'approbation du montant de la taxe sur le $\mathrm{CO}_{2}$ appliquée aux combustibles du 22 juin 2005 (FF 2005 4621).

26 L'incitation à consommer moins de combustibles induite par une telle taxe permettrait de combler 0,7 million de tonnes de $\mathrm{CO}_{2}$ de l'écart global (combustibles et carburants), estimé à 2,9 millions de tonnes de $\mathrm{CO}_{2}$ à l'horizon 2008-2012. Dernière actualisation: OFEN, Bundesratsvarianten zur Umsetzung des $\mathrm{CO}_{2}$-Gesetzes, Arbeitspapier zu Modellrechnungen auf der Basis der neuen Referenzentwicklung der Energieperspektiven des BFE, Bern, März 2005, 60 p. 
terme de laquelle, si ses effets en termes de réduction des émissions de $\mathrm{CO}_{2}$ ne sont pas suffisants, une taxe sur l'essence sera introduite. Le 30 août 2005, la Fondation centime climatique $(\mathrm{FCC})^{27}$, créée quelques jours auparavant, a signé une convention avec le Conseil fédéral, réglant les objectifs, le calendrier, les modalités d'établissement des rapports et les règles de coordination qu'elle s'engage à respecter. L'objectif est de réduire les émissions de $\mathrm{CO}_{2}$ d'au moins 1,8 million de tonnes par an à l'horizon 2008-2012. Avec les recettes du centime climatique, prélevées depuis le $1^{\text {er }}$ octobre 2005 et estimées à environ 100 millions de francs par an, la fondation va mettre en œuvre des projets en Suisse (en complément de ceux du programme SuisseEnergie et de ceux des cantons) mais surtout acheter des certificats d'émissions de $\mathrm{CO}_{2}$ à l'étranger. L'Ordonnance régissant l'imputation des réductions d'émissions opérées à l'étranger ${ }^{28}$ limite ces achats à 1,6 million de tonnes de $\mathrm{CO}_{2}$ par an. La fondation achètera ses premiers certificats d'émissions de $\mathrm{CO}_{2}$ en 2006, avec une préférence pour ceux générés par des projets utilisant des sources d'énergie renouvelable. Des certificats de réduction des émissions provenant de projets impliquant d'autres gaz à effet de serre que le $\mathrm{CO}_{2}$ sont aussi envisagés.

Fin 2005, l'Office fédéral de la statistique a produit un nouvel outil pilote ${ }^{29}$, qui relie les données principales sur les émissions de gaz à effet de serre aux données économiques émanant de la comptabilité nationale, par branche d'activités. Cet outil a permis de mettre en lumière le découplage progressif entre les émissions de gaz à effet de serre et la croissance économique en Suisse, mais également la responsabilité importante des transports quant à ces émissions. Les besoins croissants de mobilité des ménages suisses ont par exemple induit une augmentation de $16 \%$ des émissions entre 1990 et 2002.

\subsubsection{Programme SuisseEnergie}

Le programme SuisseEnergie, lancé en 2001 à la suite du programme Energie 2000, est une composante de la politique suisse en matière de climat, en liaison avec les mesures prises dans le cadre de la Loi sur le $\mathrm{CO}_{2}$ (voir plus haut), mais constitue également une pièce de la politique suisse de l'énergie ${ }^{30}$. Chaque année, le programme fait l'objet d'un rapport qui présente les moyens mis en œuvre pour atteindre les objectifs fixés et l'état d'avancement du programme dans la réalisation de ces derniers.

Selon le dernier rapport disponible ${ }^{31}$, la consommation d'énergie fossile serait de $7,6 \%$ plus élevée et les émissions de $\mathrm{CO}_{2}$ seraient de 2,8 millions de tonnes plus importantes si les programmes Energie 2000 et SuisseEnergie n'avaient pas existé.

27 Le secrétariat de la FCC est établi à Zurich depuis octobre 2005 : <www.stiftungklimarappen.ch>.

28 Conseil fédéral, Ordonnance régissant l'imputation des réductions d'émissions opérées à l'étranger (Ordonnance sur l'imputation du $\mathrm{CO}_{2}$ ) du 22 juin 2005 (RO 2005 3581).

29 Office fédéral de la statistique, Emissions de gaz à effet de serre par branche économique. NAMEA pilote pour la Suisse en 2002, 2005, 74 p.

30 Le programme vise un apport accru des énergie renouvelables dans l'offre d'énergie et une limitation de la croissance de la demande d'électricité.

31 OFEN, En marche vers l'étape suivante... 4e Rapport annuel SuisseEnergie 2004/2005, Berne, octobre $2005,39 \mathrm{p}$. 
Programmé jusqu'en 2010, SuisseEnergie est entré dans sa deuxième étape en 2005 , orientée vers «davantage de résultats et d'utilité ${ }^{32}$, selon le titre de la stratégie définie dans ce but. Cette dernière poursuit les objectifs initiaux du programme, en termes de réduction des émissions de $\mathrm{CO}_{2}$ pour la politique climatique ainsi qu'en termes de frein à l'accroissement de la demande d'électricité et de promotion des énergies renouvelables pour la politique énergétique. La stratégie prévoit, pour améliorer l'efficacité de SuisseEnergie, que ses moyens soient affectés exclusivement à cinq domaines préférentiels ${ }^{33}$.

Pour ce faire, la stratégie identifie trois principes auxquels le programme SuisseEnergie devra se tenir, soit le renforcement des partenariats entre la Confédération et les cantons, l'économie et les grandes associations, la formulation claire des objectifs de ces partenaires ainsi que la création de plates-formes de communication entre les cinq domaines prioritaires évoqués ci-dessus.

\section{SOURCES}

International Institute for Sustainable Developement (IISD), «Summary of the Eleventh Conference of the Parties to the UN Framework Convention on Climate Change and First Conference of the Parties Serving as the Meeting of the Parties to the Kyoto Protocol: 28 November-10 December 2005», Earth Negotiations Bulletin, vol. 12, n 291, 12 December 2005, 20 p.

Confédération suisse, Quatrième communication nationale de la Suisse à la CCNUCC. Première communication nationale en application du Protocole de Kyoto. 2005. Synthèse, Berne, OFEFP, 2005, 25 p., disponible sur le site Internet de l'Office fédéral de l'environnement, <www.environnement-suisse.ch>.

\subsection{Convention sur la diversité biologique}

En 2005, seul le Protocole de Cartagena a fait l'objet d'une réunion de ses Etats parties, la Convention sur la diversité biologique dont il dépend ne tenant conférence que tous les deux ans. La réunion a surtout porté sur les mouvements transfrontières des organismes génétiquement modifiés (OGM) qui, s’ils ne sont pas contrôlés, font encourir des risques à la biodiversité. Les surfaces cultivées en OGM augmentent depuis 1995 et même si les pays développés en possèdent toujours les deux tiers, c'est de plus en plus dans les pays en développement qu'elles se multiplient. En Suisse, un moratoire de cinq ans sur la culture d'OGM dans l'agriculture a été accepté par le peuple et les cantons en novembre 2005.

\subsection{1. $2^{\mathrm{e}}$ Réunion des parties au Protocole de Cartagena (Montréal, 30 mai-3 juin 2005)}

Les Etats parties au Protocole de Cartagena pour la prévention des risques biotechnologiques, au nombre de 129 en novembre 2005, se sont réunis pour la deuxième fois entre le 30 mai et le 3 juin 2005 à Montréal (COP/MOP-2). La Convention sur la diversité biologique, à laquelle ce protocole est rattaché, est l'objet d'une conférence des parties qui est organisée tous les deux ans, la prochaine étant programmée en mars 2006 au Brésil.

32 OFEN, SuisseEnergie, $2^{e}$ étape. Davantage de résultats et d'utilité. La stratégie de SuisseEnergie en 2006-2010, Berne, 23 juin 2005, 49 p.

33 La modernisation des bâtiments; les énergies renouvelables; les moteurs et appareils à bon rendement énergétique; l'utilisation rationnelle de l'énergie et la récupération de chaleur dans l'industrie; la mobilité peu gourmande d'énergie et peu polluante. 


\section{Situation des cultures d'organismes génétiquement modifiés dans le monde en 2004}

Les surfaces de cultures d'organismes génétiquement modifiés sont 47 fois plus importantes en 2004 qu'en 1996, soit un an après leur première apparition. Elles couvraient, en 2004, 81 millions d'hectares, soit plus d'une fois et demie la surface de la France, contre 67,7 millions d'hectares en 2003. Les principales plantes génétiquement modifiées sont le soja (48,4 millions d'hectares en 2004), le maïs (19,3), le coton (9) et le colza $(4,3)$. Les surfaces de soja transgénique représentent $56 \%$ du total des surfaces mondiales plantées en soja, ce chiffre étant de $14 \%$ pour le maïs, $28 \%$ pour le coton et $19 \%$ pour le colza.

En 2004, 17 pays possédaient des cultures d'organismes génétiquement modifiés, mais $99 \%$ des surfaces ainsi cultivées se situent dans seulement huit pays, les Etats-Unis (59\%), l'Argentine (20\%), le Canada (6\%), le Brésil (6\%), la Chine (5\%), le Paraguay (2\%), I'Inde et l'Afrique du Sud (chacune environ $1 \%$ ). Par rapport au total mondial, la part des surfaces plantées en organismes transgéniques dans les pays en développement est de 34\%, avec une augmentation de ces dernières qui, entre 2003 et 2004, a pour la première fois dépassé celle enregistrée dans les pays industrialisés (7,2 millions d'hectares dans les PVD contre 6,1 millions dans les pays industrialisés).

Les autres pays cultivant des organismes génétiquement modifiés sont, par ordre d'importance, I'Uruguay, I'Australie, la Roumanie, le Mexique, l'Espagne, les Philippines, la Colombie, le Honduras et l'Allemagne. Ces trois derniers pays possèdent des surfaces inférieures à 50 '000 hectares.

Source: Clive James, Preview: Global Status of Commercialized Biotech/GM Crops: 2004, ISAAA Briefs, nº 32, Ithaca, NY, ISAAA, 2004, 12 p.

En 2004, la $1^{\text {re }}$ Réunion des parties au Protocole de Cartagena avait institué un Groupe spécial d'experts sur la responsabilité et la réparation des dommages occasionnés par les mouvements transfrontières des organismes vivants modifiés $(\mathrm{OVM})^{34}$. Ce groupe s'est réuni pour la première fois du 25 au 27 mai 2005 et a travaillé à l'élaboration d'une réglementation internationale en matière de responsabilité et de réparation, qui doit voir le jour en 2007.

Le thème principal de l'ordre du jour de la $2^{\mathrm{e}}$ Réunion des parties au protocole $\mathrm{a}$ été d'élaborer des exigences détaillées pour la documentation devant accompagner les mouvements transfrontières des organismes vivants modifiés destinés à l'alimentation humaine et animale ou à la transformation. Selon le protocole, les Etats parties avaient en effet deux ans, depuis son entrée en vigueur en 2003, pour s'accorder sur ces exigences. Malgré cette date butoir, le consensus n'a pu être trouvé, le Brésil et la Nouvelle-Zélande ne pouvant se rallier au texte de compromis. Ce texte avait été proposé par le Suisse François Pythoud, de l'OFEFP, qui coprésidait le groupe de travail chargé de traiter, entre autres, cette question de la documentation. Les divisions étaient marquées entre les pays exportateurs et les pays importateurs d'OVM, pour qui une documentation mentionnant «contenant des OVM» ou «pouvant contenir des OVM»n'a pas la même signification. De plus, la vérification de cette documentation est liée aux travaux du Groupe spécial d'experts travaillant sur un régime international de responsabilité et de réparation. «En effet, si un régime plus strict était mis en place, alors la validité des revendications concernant la documentation pourrait être amoindrie. De même, si le respect des dispositions du protocole est suivi de près et appliqué de manière stricte, alors des dispositions faibles en matière de

34 Dans la convention et le protocole, la terminologie d' «organisme vivant modifié» (OVM) est préférée à celle d' «organisme génétiquement modifié»(OGM), bien que cette dernière soit plus connue du grand public. 
documentation auraient un impact moins significatif sur la protection de la biodiversité. $\gg^{35}$

La question de la documentation sera reprise lors de la prochaine Réunion des parties au Protocole de Cartagena, sur la base de la proposition de compromis suisse. Malgré son rôle remarqué sur cette question et le bilan positif qu'elle tire de toute la réunion, la Suisse considère que les perspectives d'avancées à la $3^{\mathrm{e}}$ Réunion des parties en 2006 sont limitées. A l'instar de l'Union européenne, elle a ancré des dispositions contraignantes sur l'identification des OVM dans sa législation nationale ${ }^{36}$ et ce modèle apparaît progressivement à de nombreux pays en développement comme le seul moyen de se protéger contre d'éventuels accidents lors des mouvements transfrontières de tels organismes.

Malgré cet échec, des progrès ont été accomplis dans les autres domaines de discussions, notamment sur l'évaluation et la gestion des risques biotechnologiques, sur le renforcement des capacités et sur la conscientisation et la participation du public face au transfert, à la manipulation et à l'utilisation des OVM.

Pour la Suisse, deux questions importantes ont émergé lors de cette $2^{\mathrm{e}}$ Réunion des parties, celle des droits et des obligations des pays de transit des OVM et celle du rôle de la recherche publique en matière d'OVM.

\subsubsection{Agriculture suisse et organismes génétiquement modifiés}

En décembre 2003, l'Office fédéral de l'agriculture avait mandaté la Station fédérale de recherches en agroécologie et agriculture (Agroscope) pour évaluer les mesures permettant la coexistence des productions agricoles avec et sans OGM en Suisse. Cette étude, lancée afin de permettre une prise de position du Conseil fédéral sur l'Initiative «pour des aliments produits sans manipulations génétiques», est arrivée à son terme en avril 2005. Le rapport conclut qu' «à condition de prendre des mesures techniques et organisationnelles dans la pratique agricole et de se mettre d'accord pour respecter des distances d'isolement, la coexistence de systèmes culturaux ayant ou non recours au génie génétique est possible en Suisse du point de vue scientifique ${ }^{37}$. La Loi sur le génie génétique, en vigueur depuis le $1^{\text {er }}$ janvier 2004, repose sur le principe que les OGM ne peuvent être utilisés que s'ils sont autorisés et seulement s'ils n'affectent pas la production sans OGM. Pour la mise en œuvre de ces obligations, un projet d'ordonnance sur la coexistence ${ }^{38}$ a été mis en consultation le 3 octobre 2005 par le Département fédéral de l'économie, accompagné d'un commentaire.

35 Institut international du développement durable (IIDD), «Résumé de la première réunion du Groupe spécial sur la responsabilité et la réparation et deuxième réunion des parties au Protocole de Cartagena pour la prévention des risques biotechnologiques. 25 mai-3 juin 2005 », Bulletin des négociations de la Terre, vol. 9, no 320, 6 juin 2005, p. 15.

36 Conseil fédéral, Ordonnance sur les mouvements transfrontières des organismes génétiquement modifiés (Ordonnance de Cartagena, OCart) du 3 novembre 2004 (RS 814.912.21, RO 2004 4801).

37 Agroscope FAL Reckenholz, Possible coexistence de cultures agricoles traditionnelles et génétiquement modifiées, communiqué de presse, 25 avril 2005, p. 1, disponible sur le site Internet de la station fédérale, <www.reckenholz.ch>.

38 Ordonnance sur les mesures de coexistence lors de la culture de plantes génétiquement modifiées et lors de l'utilisation du produit de la récolte (Ordonnance sur la coexistence). 
L'Initiative populaire «pour des aliments produits sans manipulations génétiques» demande l'introduction d'une disposition transitoire dans la Constitution fédérale, stipulant que l'agriculture suisse n'utilise pas d'organismes génétiquement modifiés pendant les cinq ans qui suivent l'adoption par le peuple et les cantons de cette disposition. Cette initiative avait abouti en octobre 2003 puis été soumise au Parlement par le Conseil fédéral en août 2004. En juin 2005, les Chambres fédérales ont recommandé au peuple de la rejeter, suivant en cela le message du Conseil fédéral ${ }^{39}$.

[D] Annuaire 2005, $\mathrm{n}^{\circ}$ 1, moratoire sur l'utilisation des OGM dans l'agriculture suisse, pp. 168-169.

Les arguments pour ou contre l'initiative étaient connus depuis le rejet du Conseil fédéral en 2004 et ont pratiquement été repris tels quels dans les campagnes qui ont précédé la votation. Les partis politiques de droite (PDC, PRD, PLS et UDC) rejetaient majoritairement le moratoire, de même qu'economiesuisse, au nom surtout du frein à la croissance et à la recherche qu'il provoquerait. La gauche était en faveur de ce moratoire, de même que les paysans suisses, y compris ceux de l'UDC, défendant le principe de précaution sur lequel il se basait. Les sondages prédisaient une large victoire du camp en faveur de l'initiative. Le 27 novembre 2005, l'initiative a été acceptée par le peuple à $55,7 \%$ des votants et à l'unanimité des cantons. Le moratoire est donc en vigueur jusqu'au 27 novembre 2010, bien qu'aucune demande d'homologation de semences génétiquement modifiées pour leur utilisation dans l'agriculture suisse n'eût été déposée jusqu'alors. Suite à cette décision du peuple suisse contraire à sa recommandation, le Conseil fédéral a décidé, le 2 décembre 2005, de lancer un nouveau programme national de recherche qui sera réalisé par le Fonds national suisse de la recherche scientifique. Doté de 12 millions de francs, le programme «utilité et risques de la dissémination de plantes génétiquement modifiées» doit préparer les bases décisionnelles du débat politique qui aura indubitablement lieu à l'échéance du moratoire.

\subsubsection{Révision de la loi sur les brevets: message du Conseil fédéral}

L'origine du processus de modification de la Loi fédérale sur les brevets d'invention (LBI) remonte à la motion Leumann du 10 juin $1998^{40}$, chargeant le Conseil fédéral d'harmoniser les dispositions de cette loi avec la Directive européenne sur la biotechnologie ${ }^{41}$, cela également dans le prolongement du projet Gen-Lex.

Annuaire 2001, projet Gen-Lex et révision de la loi sur les brevets, pp. 208-211.

La question de la délivrance de brevets portant sur les inventions biotechnologiques reste centrale dans la dernière version du projet de révision de la LBI mais ce dernier doit aussi permettre «la transposition en Suisse de la résolution du Conseil général de l'Organisation mondiale du commerce du 30 août 2003 visant à procurer aux pays en développement un meilleur accès aux

39 Conseil fédéral, Message concernant l'Initiative populaire "pour des aliments produits sans manipulations génétiques » du 18 août 2004 (FF 2004 4629).

40 Motion Helen Leumann du 10 juin 1998 (98.3243).

41 Directive 98/44/CE du Parlement européen et du Conseil du 6 juillet 1998 relative à la protection juridique des inventions biotechnologiques, Journal officiel des Communautés européennes (JOCE), $\mathrm{n}^{\mathrm{o}} \mathrm{L} 213,30$ juillet 1998, pp. 13-21. 
médicaments brevetés $\gg^{42}$ et de prendre en compte les développements récents, en Suisse et sur le plan international, en matière de protection de la propriété intellectuelle.

La première procédure de consultation portant, entre autres, sur le projet de révision de la LBI s'est déroulée de décembre 2001 à avril 2002 et a montré que le sujet était trop technique et complexe pour pouvoir être soumis en l'état au Parlement. Une deuxième procédure de consultation a été organisée de juin à octobre 2004, sur une version remaniée du projet de révision de la LBI, basée sur un long travail d'enquête et de discussions mené auparavant par l'Institut fédéral de la propriété intellectuelle. Le Département fédéral de justice et police (DFJP) a produit un rapport sur les résultats de cette procédure de consultation en janvier $2005^{43}$.

En mars 2005, le Conseil fédéral a chargé le DFJP d'élaborer un nouveau projet de révision de la LBI et décidé d'échelonner la révision de la LBI, selon la portée de ses différents volets et l'urgence à les mettre en œuvre. L'approbation de deux traités internationaux relatifs au système du brevet européen a ainsi fait l'objet d'un message du Conseil fédéral, le 18 mai 2005 déjà ${ }^{44}$. Le Conseil des Etats a adopté les deux arrêtés fédéraux touchant à l'approbation de ces traités le 5 octobre 2005. Si le Conseil national suit, l'approbation de ces traités appellera une adaptation de la LBI.

Le 23 novembre 2005, le Conseil fédéral a soumis aux Chambres son Message concernant la modification de la Loi sur les brevets et l'Arrêté fédéral portant approbation du Traité sur le droit des brevets et du règlement d'exécution ${ }^{45}$. Ce message sera présenté dans l'Annuaire suisse de politique de développement 2007, avec le débat aux Chambres fédérales sur le projet de modification de la LBI.

\section{SOURCES}

Institut international du développement durable (IIDD), «Résumé de la première réunion du Groupe spécial sur la responsabilité et la réparation et deuxième réunion des parties au Protocole de Cartagena pour la prévention des risques biotechnologiques. 25 mai-3 juin 2005 », Bulletin des négociations de la Terre, vol. 9, n 320, 6 juin 2005, 16 p.

Conseil fédéral, Message concernant la modification de la Loi sur les brevets et l'Arrêté fédéral portant approbation du Traité sur le droit des brevets et du règlement d'exécution (FF 2006 1).

\subsection{Forêts}

Les forêts, qu'elles soient tropicales, boréales ou tempérées, sont centrales dans toutes les problématiques d'environnement et de développement, en termes de biodiversité (pour les espèces et les populations qu'elles abritent), en termes de

42 Conseil fédéral, Message concernant la modification de la Loi sur les brevets et l'Arrêté fédéral portant approbation du Traité sur le droit des brevets et du règlement d'exécution (FF 2006 1), p. 11.

43 Département fédéral de justice et police, Modification du droit des brevets. Rapport sur les résultats de la procédure de consultation, Berne, janvier 2005, 62 p., disponible sur le site Internet de l'Institut fédéral de la propriété intellectuelle, <www.ipi.ch $>>$ ige $>$ infos juridiques $>$ domaines juridiques >brevets.

44 Conseil fédéral, Message concernant l'approbation de deux traités relatifs au système du brevet européen et la modification de la Loi sur les brevets du 18 mai 2005 (FF 2005 3569).

45 Op. cit. 
climat (pour les puits de carbone qu'elles constituent) et dans une perspective de développement durable (les forêts sont plus que les produits commerciaux du bois). Cette centralité exige que les objectifs de commercialisation des ressources forestières et de conservation de la forêt aillent de pair. Un accord international sur les bois tropicaux existe depuis 1985 mais les efforts visant à le renégocier sont restés vains en 2005. En ce qui concerne l'arrangement international relatif à tous les types de forêts, il n'est juridiquement pas contraignant, ce qu'a tenté de changer la Suisse en 2005, mais sans succès. Une piste pour relier les deux objectifs ci-dessus est peut-être de labelliser le bois, par exemple au travers du Forest Stewardship Council (FSC).

\subsubsection{Accord international sur les bois tropicaux}

L'Accord international sur les bois tropicaux (AIBT) a été négocié sous l'égide de la Conférence des Nations unies sur le commerce et le développement (CNUCED) puis conclu à Genève en novembre 1983. Il est entré en vigueur en avril 1985, la Suisse l'ayant ratifié le 9 mai de la même année. L'enjeu de l'accord a été et demeure aussi bien la préservation et la mise en valeur de la forêt tropicale que le commerce du bois issu de cette forêt. Cet accord «anticipait de fait les problématiques développées dans le Rapport Brundtland de 1987 et au Sommet de la Terre de 1992, et ses éléments relatifs au commerce, outre leurs finalités propres, n'en étaient pas moins des instruments au service de la conservation des forêts tropicales ${ }^{46}$. L'AIBT inclut en effet plusieurs références au but de l'utilisation durable des forêts.

L'Organisation internationale des bois tropicaux (OIBT) a été créée par l'AIBT en 1986, sous les auspices des Nations unies. Son secrétariat est à Yokohama, au Japon. La mission de l'OIBT est de faciliter les échanges d'informations, les consultations et la coopération entre ses membres quant au commerce international des bois tropicaux et à la gestion durable de leur base de ressources. L'OIBT vise ainsi à promouvoir le commerce de bois tropical provenant de forêts exploitées de manière durable. En outre, l'OIBT assiste financièrement des projets présentés par les pays membres. Ceux-ci sont au nombre de 59 (état en décembre 2005), répartis en deux caucus, le premier rassemblant les 33 pays producteurs de bois tropicaux et le second les 26 pays consommateurs (dont la Suisse). En termes de représentativité, les quatre cinquièmes de l'ensemble des forêts tropicales se situent dans les pays membres de l'OIBT et $90 \%$ du commerce total de bois tropicaux se fait entre eux.

Au cours de son histoire, l'OIBT s'est dotée de plans d'action permettant de réaliser son objectif 2000, soit de viser à ce que les exportations de bois tropicaux et de produits dérivés soient le plus tôt possible issues de ressources en gestion durable. En conséquence, l'AIBT a été régulièrement remis en négociation et remplacé par un nouvel accord reflétant les engagements et ambitions des pays membres de l'OIBT. En 2005, les principales questions qui ont occupé les délégations dans la renégociation de l'accord ont été de redéfinir ses objectifs (intégrer dans ceux-ci les activités de l'OIBT dans les domaines des produits forestiers non ligneux et des services écologiques rendus par les forêts

46 International Tropical Timber Organization (ITTO), About ITTO, p. 1, disponible sur le site de l'OIBT, <www.itto.or.jp>. 
tropicales) et de réformer l'arrangement administratif et financier de l'OIBT (essentiellement l'augmentation du budget, la répartition des contributions et des voix entre les pays ainsi que le rythme des réunions). Jusqu'à fin 2005, toutes les sessions de la Conférence des Nations unies pour la renégociation de l'accord se sont soldées par des échecs. En janvier 2006, les négociations ont cependant été conclues avec succès.

\subsubsection{Forum des Nations unies sur les forêts}

La question des forêts avait été parmi les sujets de discussion les plus controversés de la Conférence des Nations unies sur l'environnement et le développement, tenue à Rio en 1992. Elle avait opposé les pays développés aux pays en développement, les premiers désirant une convention sur les forêts tropicales seulement, alors que les seconds défendaient l'idée d'une convention sur tous les types de forêts. En conséquence, la convention sur les forêts n'a pas vu le jour lors de la conférence de Rio et c'est une Déclaration de principes, non juridiquement contraignante mais faisant autorité, pour un consensus mondial sur la gestion, la conservation et l'exploitation écologiquement viable de tous les types de forêts ${ }^{47}$ qui en était ressortie.

Depuis, au sein des Nations unies, des efforts considérables ont été déployés dans le sens de cette déclaration, d'abord avec la création, en 1995, du Panel (devenu Forum en 1997) intergouvernemental sur les forêts sous les auspices de la Commission du développement durable, puis avec l'établissement par l'ECOSOC, en 2000, d'un organe subsidiaire intergouvernemental permanent, nommé Forum des Nations unies sur les forêts (FNUF).

En même temps que cet organe permanent, l'ECOSOC a également établi un Arrangement international sur les forêts ${ }^{48}$. Le FNUF et l'arrangement international partagent le même objectif principal: promouvoir la mise en œuvre des propositions d'actions volontaires adoptées par le Panel et le Forum intergouvernemental sur les forêts et «obtenir un engagement politique à long terme en vue de promouvoir la gestion, la conservation et le développement durable de tous les types de forêts $»^{49}$. Dans sa première session, en juin 2001, le FNUF s'est doté d'un plan d'action non contraignant et d'un programme de travail indiquant les thèmes qui seraient examinés lors de chaque nouvelle session annuelle. Pour assister le FNUF dans sa fonction, le Partenariat de collaboration sur les forêts a été créé en avril 2001. Fin 2005, ce partenariat regroupait 14 membres $^{50}$.

La 5e Session du FNUF s'est déroulée du 16 au 27 mai 2005 à New York. L'objectif principal de cette session était d'évaluer l'efficacité de l'Arrangement

47 Assemblée générale des Nations unies, Rapport de la CNUE (Rio de Janeiro, 3-14 juin 1992), Annexe III, doc. A/CONF.151/26 (Vol. III), p. 124. L'Agenda 21 comprend également un chapitre relatif aux forêts (chapitre 11 : «Lutte contre le déboisement»).

48 Cet arrangement est issu de la 4 e Session du Forum intergouvernemental sur les forêts: Conseil économique et social des Nations unies, Rapport du Forum intergouvernemental sur les forêts sur sa quatrième session, New York, 31 janvier-11 février 2000, Appendice, doc. E/CN.17/2000/14, 20 mars 2000, pp. 44-46.

49 Ibid., p. 44.

50 Centre pour la recherche forestière internationale (CIFOR), Organisation des Nations unies pour l'alimentation et l'agriculture (FAO), Organisation internationale des bois tropicaux (OIBT), Union internationale des instituts de recherche forestière (IUFRO), Secrétariat de la Convention sur la 
international sur les forêts, cinq ans après son élaboration, et de prendre une décision quant à son futur. La grande question qui était restée en suspens à l'époque était l'opportunité de créer un instrument juridique contraignant pour réaliser l'objectif principal du FNUF énoncé ci-dessus. La Suisse a défendu la mise en place d'un tel instrument, qui pourrait établir un cadre institutionnel de coopération et ainsi générer les ressources financières additionnelles réclamées par les pays en développement. En préparation à cette $5^{\text {e }}$ Session du FNUF, la Suisse a d'ailleurs lancé un processus informel dit «des amis du FNUF» en faveur d'un arrangement international sur les forêts alternatif, renforçant le FNUF. Des solutions ont été identifiées lors de ce processus, notamment pour une formulation d'objectifs quantifiables et pour une approche plus régionale du travail du FNUF. La session s'est terminée sans qu'aucun accord n'ait pu être finalisé mais, en février 2006, la 6e Session du FNUF a débouché sur un accord pour le renforcement de l'Arrangement international sur les forêts.

\subsubsection{Le label du bois octroyé par l'organisation Forest Stewardship Council (FSC)}

En 2005, la déforestation a atteint les deux tiers de la surface totale des forêts du globe et la poursuite de ce phénomène est facilitée par l'absence de protection caractérisant $90 \%$ de la trentaine de millions de kilomètres carrés de forêts restants. Protéger ces forêts tout en permettant leur exploitation selon des critères écologiques, sociaux et économiques est la tâche que s'est assignée le Forest Stewardship Council (FSC), organisation non gouvernementale fondée en 1993 à Toronto. Cette organisation bénéficie d'une reconnaissance internationale tant des exploitants et des transformateurs du bois que des consommateurs. Le label FSC garantit que tout le parcours du bois est conforme aux principes et critères que cette organisation exige au minimum des pays dans lesquels elle est engagée ${ }^{51}$.

\section{Surfaces de forêts certifiées FSC dans le monde, fin 2005}

Fin 2005, ce sont environ 68 millions d'hectares a de forêts répartis dans 66 pays qui étaient exploités selon les directives du FSC. A cette date, en Suisse, un tiers environ des surfaces boisées était certifié FSC, soit 427 mille hectares. Plus de $80 \%$ des forêts certifiées FSC se trouvent en Europe ly compris les pays d'Europe de l'Est) et en Amérique du Nord. L'Afrique a une surface boisée certifiée de 1,7 million d'hectares répartis dans sept pays, dont 1,4 million en Afrique du Sud. Pour l'Amérique latine, les chiffres sont de presque 8 millions d'hectares dans 17 pays, dont plus de 5,5 millions au Brésil et en Bolivie. En Asie, ce sont 2,3 millions d'hectares qui sont certifiés FSC dans 11 pays.

Par rapport à la superficie forestière mondiale, estimée à près de 4 milliards d'hectares par la $F A 0^{b}$, les surfaces de forêts certifiées ne représentent donc qu'environ 1,7\%. Pour compléter les références, il faut encore mentionner que la déforestation atteind un rythme annuel de 13 millions d'hectares alors

diversité biologique (CDB), Secrétariat du Fonds pour l'environnement mondial (FEM), Secrétariat de la Convention des Nations unies sur la lutte contre la désertification, Secrétariat du Forum des Nations unies sur les forêts (FNUF), Secrétariat de la Convention-cadre des Nations unies sur les changements climatiques (CCNUCC), Programme des Nations unies pour le développement (PNUD), Programme des Nations unies pour l'environnement (PNUE), World Agroforestry Centre (ICRAF), Banque mondiale, Union mondiale pour la nature (UICN).

51 Forest Stewardship Council, FSC Principles and Criteria for Forest Stewardship, April 2004, 11 p., disponible sur le site Internet du FSC, <www.fsc.org >. 
que la plantation et l'extention naturelle des forêts sont estimées chaque année à moins de 6 millions d'hectares par la FAO.

Les forêts tropicales ne représentent qu'un peu moins de $13 \%$ des surfaces certifiées, le reste étant partagé par les forêts tempérées (près de $43 \%$ ) et les forêts boréales (près de $45 \%$ ).

Les plantations certifiées gagnent en importance, même si les forêts naturelles certifiées représentent toujours plus de la moitié des surfaces totales certifiées (56\%). Certaines surfaces certifiées sont recouvertes de forêts naturelles appauvries revalorisées avec des plantations $(33 \%)$ et le reste est purement constitué de plantations.

Source: Information on Certified Forest Sites endorsed by FSC, <www.certified-forests.org $>$. Les chiffres de ce site Internet sont régulièrement mis à jour (dernière consultation: janvier 2006).

a 1 kilomètre carré égale 100 hectares.

b FAO, Global Forest Assessment 2005 : Progress towards Sustainable Forest Management, FAO Forestry Paper, $\mathrm{n}^{0}$ 147, Rome, 2005, 348 p., disponible sur le site Internet de la FA0, <www.fao.org

Si les filières commerciales de bois certifié gagnent peu à peu en importance, il ne faut pas sous-estimer l'exploitation illégale de bois qui, dans de nombreux pays en développement mais également en Europe orientale où elle a lieu, a des conséquences sociales, environnementales et économiques extrêmement dommageables. Avant d'entrer dans les flux commerciaux internationaux, le bois issu de ces exploitations subit une sorte de blanchiment, transitant par plusieurs pays pour y être transformé.

Une étude du WWF Suisse a estimé, pour 2003, les importations en Suisse de bois exploité de manière illégale ou douteuse à environ $8 \%$ de ses importations totales de bois $^{52} .90 \%$ de ces dernières provenaient de l'Union européenne d'alors (l'Europe des Quinze), sous forme de papier ou de meubles. La branche britannique du WWF a produit quant à elle en novembre 2005 un rapport couvrant également le commerce de bois exploité illégalement auquel l'Union européenne actuelle prend part $^{53}$. Ce rapport, en analysant les importations des pays de l'Union européenne en 2004 en provenance de six régions du monde ${ }^{54}$, a pour ambition d'évaluer la part de responsabilité de l'exploitation illégale des forêts menant à leur disparition, qui se déroule à un taux de plus de 12 millions d'hectares par an, et de mesurer l'impact d'une telle exploitation sur les conditions de vie des populations locales. Le rapport a également pour but de déterminer dans quelle mesure l'Union européenne pourrait utiliser son influence commerciale afin de limiter l'exploitation illégale du bois et ainsi de minimiser ses conséquences dramatiques d'un point de vue du développement durable.

\section{SITES INTERNET}

Forum des Nations unies sur les forêts : <www.un.org/esa/forests >.

Organisation des Nations unies pour l'alimentation et l'agriculture: <www.fao.org $>$.

Organisation internationale des bois tropicaux : <www.itto.or.jp $>$.

Forest Stewardship Council : <www.fsc.org >.

World Wild Fund for Nature: <www.wwf.org >.

52 WWF Schweiz, Illegaler Holzeinschlag und die Schweiz. Eine Analyse der Schweizer Aussenhandelsdaten 2003, März 2005, 44 p., comprenant un résumé en français.

53 WWF UK, Failing the Forests: Europe's Illegal Timber Trade, November 2005, 102 p.

54 Bassin amazonien, Pays baltes, bassin du Congo, Afrique de l'Est, Indonésie et Russie. Ces six régions procurent les trois quarts du bois illégal présent dans l'Union européenne. 